\title{
MIMO Self-Tuning Control of Chemical Process Operation
}

\author{
Hallager, L.; Jørgensen, S. B.; Goldschmidt, L.
}

Published in:

American Control Conference

Publication date:

1984

Document Version

Publisher's PDF, also known as Version of record

Link back to DTU Orbit

Citation (APA):

Hallager, L., Jørgensen, S. B., \& Goldschmidt, L. (1984). MIMO Self-Tuning Control of Chemical Process Operation. In American Control Conference (pp. 62-66). IEEE.

\section{General rights}

Copyright and moral rights for the publications made accessible in the public portal are retained by the authors and/or other copyright owners and it is a condition of accessing publications that users recognise and abide by the legal requirements associated with these rights.

- Users may download and print one copy of any publication from the public portal for the purpose of private study or research.

- You may not further distribute the material or use it for any profit-making activity or commercial gain

- You may freely distribute the URL identifying the publication in the public portal

If you believe that this document breaches copyright please contact us providing details, and we will remove access to the work immediately and investigate your claim. 


\section{WA3 - 11:15}

MIMO Self-Tuning Control of Chemical Process Operation

L. Hallager, 5.B. Jorgensen, and L. Goldschmidt

Dept. of Chemical Engineering, Technical University of Denmark Building 229, DK-2800 Lyngby, Denmark

\section{Abstract}

The problem of selecting a feasible model structure for a MIMD self-tuming controller (MIMOSC) is addressed. The dependency of the necessary structure complexity in relation to the specific process operating point is investigated. Experimental results from a fixed-bed chemical reactor are used to illustrate the points.

Keywords. Adaptive control, process dynamics, chemical reactor control, process identification, recursive parameter estimation, quadratic optimal control.

\section{Introduction}

Chemical engineering plants contain in general a number of energy and mass exchange processes combined with chemical reaction processes. In either type of process at least two streams or components are brought into close (direct or indirect) contact whereupon the exchange or reaction occurs. The processes are most often carried out in tubular units or equipment with many stages, hence the processes tend to have distributed character. In addition the processes are bilinear and often nonlinear due to the thermodynamic or reaction kinetic relationships. In industrial practice slow changes often occur in internal process characteristics such as dirt deposition on heat exchange surfaces and catalyst or biological activity changes. The control problems for chemical processes are mainly to maintain product quality within specified limits and if that fails then to maintain production if at all possible. In principle these control problems are multivariable due to the number of streams or components involved. Traditionally, however, they have often been solved as multi loop problems. This solution has been satisfactory in the vast majority of the cases. However, a number of processes have remained difficult to control thus requiring a multivariable design technique. With the increased tendency towards process intensification many more control problems may advantageously be solved while recognizing their multivariable nature.

During the last decade it has been demonstrated experimentally that many chemical engineering processes often can be satisfactorily controlled using multivariable techniques.
The control designs applied generally have been based upon off line identified linearized mathematical models. These design methods, however, have not been applied significantly in process control practice; probably due to the effort required to develop a mathematical model from basic physics and chemistry using conservation principles. Furthermore, it is often difficult and costly to identify such a model, partly because estimates of some parameters may be difficult to obtain from experiments, and partly because the model will often be nonlinear in the parameters. Finally, the parameters in chemical processes are usually time varying, for instance due to changes in internal process characteristics thus necessitating more or less regular updating of parameters. Consequently, there are obvious opportunities for multivariable adaptive control design methods.

The method presented in this paper is based upon an indirect or explicit approach where simple linear relationships are postulated between measurements and control inputs. The relationships are based upon a priori chemical engineering process knowledge concerning bulk flow directions, energy and mass transfers, and rough estimates of the time delays involved. By estimating the coefficients of these relationships on line it is possible for the model to adapt to slow changes either in internal process characteristics or in external loads. The appropriate feedback gains are evaluated based upon the identified model using some specific control strategy. Relatively few investigators have investigated algorithms for tuning of controllers in the multivariable case. Peterka and Aström (1973) proposed a multivariable selft-tuning regulator based on linear quadratic optimal control of processes with uncertain parameters. Borison (1975, 1979) extended the basic minimum variance self-tuning controller to the multivariable case. Keviczky and Hetthéssy (1977) used a dead time transformation. Koivo (1980) extended Clarke and Gawthrop's method to a MIMO self-tuning controller with an equal number of inputs and outputs. Prager and Wellstead (1980) use a pole placement design procedure. Buchholt and Kümel (1981) investigated a one step control method on a two stage evaporator. Bayoumi et al. (1982) presented an algorithm for model order determination and considered processes where the time delay is the same for all control signals. 
In chemical engineering processes it is often advantageous to use more measurements than controls. An important feature of the algorithm developed herein is that it does not require the number of measurements to be equal to the number of controls. This requirement is also fulfilled by the algorithms presented by Grimble and Moir (1983).

In the multivariable case the selection of a proper model structure is very important in order to reduce the computer work-load and numerical problems. The emphasis in this presentation is on differences in the model structure arising from different process operating points.

Parameter estimation is performed using a recursive extended least squares method with variable forgetting factor. Controller gains are calculated using deterministic optimal control law with integral effect. The two latter subjects are described in Hallager and Jergensen (1983), Hallager (1984), and Hallager et al. (1984).

\section{Experimental Process}

A chemical reactor is used as an example of a typical chemical engineering process. The pilot plant reactor considered consists of a tubular fixed bed of catalyst particles, designed to be essentially adiabatic and with negligible heat capacity of the reactor wall. It is described in detail by Hansen and Jorgensen (1976). In this series of experiments a stream of hydrogen containing 0.25-0.75 mole \% oxygen is fed to the packed catalyst bed at a temperature of approximately $82^{\circ} \mathrm{C}$. The steady state total flow rate is $2.6 \mathrm{mg} / \mathrm{cm}^{2} / \mathrm{sec}$. The adiabatic temperature rise during water formation is $169^{\circ} \mathrm{C}$ per mole \% reacted oxygen.

The propagation of inlet temperature disturbances is illustrated in Figs. $l a$ and $1 b$. The open loop temperature responses at selected axial positions are shown subject to an inlet temperature pulse disturbance of height $5^{\circ} \mathrm{C}$ and duration 12 min. at low ( 0.25 mole $\%$ ) and medium ( 0.75 mole \%) inlet oxygen concentration respectively. At the low oxygen concentration ( $F$ ig. Ia) the process behaves very much as a delay with dispersion and approximately unity gain, whereas the gain increases and significant inverse response occurs when the oxygen concentration level is raised to 0.75 mole \% ( $F$ ig. lb).

\section{Qualitative Dynamical Description}

In the gas phase fixed bed catalytic reactor, investigated here, the heat capacity of the gas is negligible compared to the heat capacity of the catalyst pellets, thus the thermal residence time is several hundred times greater than the gas residence time. Furthermore, it is known (Hansen, 1973) that the mass transport processes are sufficiently fast to make quasi stationary descriptions of the mass-balances reasonable. Finally, there is almost thermal equilibrium between the catalyst pellets and the gas and within the pellets. A reasonable simplified mathematical model of the reactor therefore is a pseudo homogeneous model consisting of one dynamic nonlinear partial differential equation for the temperature and one quasi stationary differential equation for the oxygen balance. Both including dispersive terms.
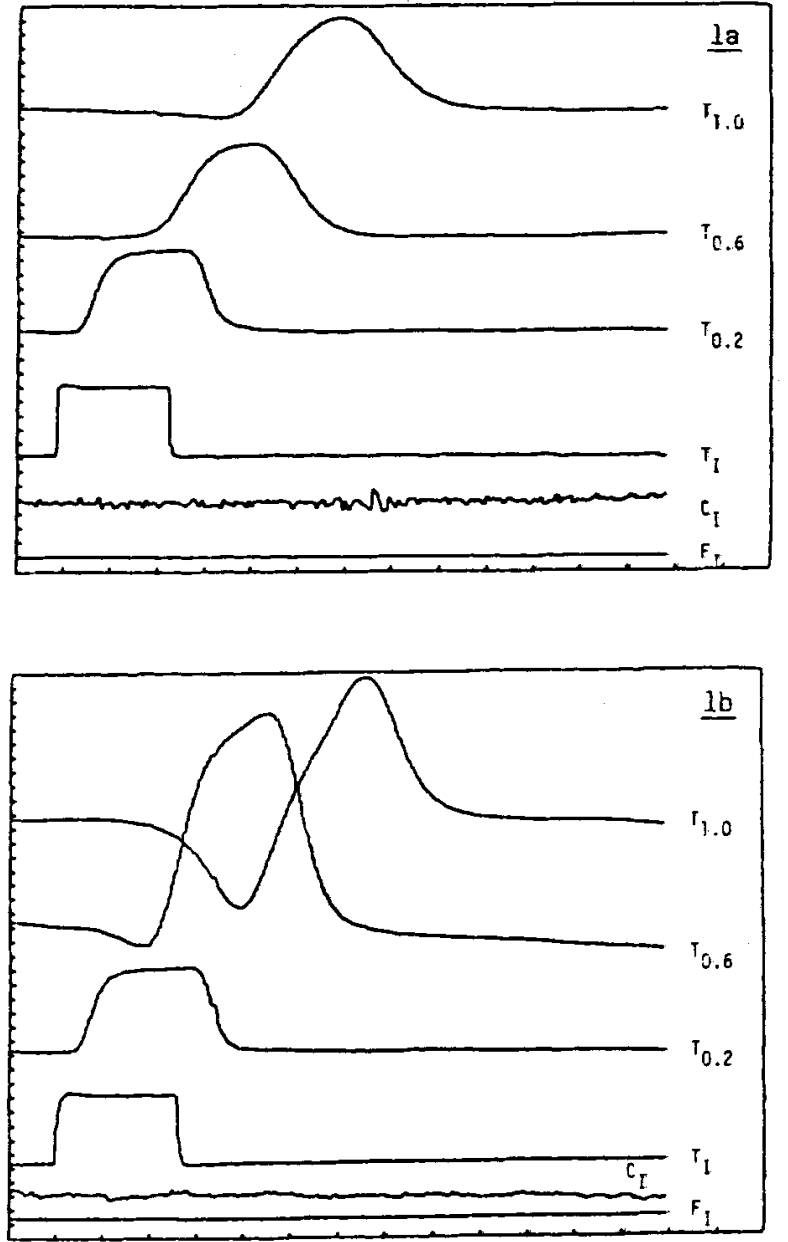

\section{Fiqure 1:}

Open loop responses of selected axial reactor temperatures and inlet variables subject to an inlet temperature pulse disturbance of height $5^{\circ} \mathrm{C}$ and duration $12 \mathrm{~min}$. at inlet oxygen concentrations of a: 0.25 and b: 0.75 mole \%. The fimensionless axial measurement positions are indicated with subscript.

The two important dynanical features of the reactor system are thus,

a. A fast propagation of concentra "am and flow rate changes. The mass balance is: a quasi stationary functional of the inlet conditions and the temperature profile.

b. A slow propagation of temperature changes. The thermal wave passes through the reactor in 1130 minutes dependent upon the flaw rate.

The qualitative process knowledge discussed above may be presented in a diagram which will be useful in selection of model structure and control strategy. The necessary ingredients are:

i) The sufficient number of conserved quantities: momentum, mass, and energy necessary to describe the process state with a for practical 
purposes reasonable accuracy.

ii) The significant capacities.

iii) The quantity transport mechanisms.

iv) The presence of couplings among the quantities.

For many chemical engineering processes the Dynamic Quantity Interactions and flows in the process may be depicted clearly in a Diagram (a DYQUID) as shown in Figure 2 for the example process. The energy quantity having a significant capacity is shown as a box which is rectangular in order to indicate the axial coordinate of the reactor. The total mass and oxygen quantities are shown as horizontal lines due to their relatively low capacities.

The purpose of the DVQUID is in this context to enable a reasonable selection of:

i) Type and position of measurements.

ii) Controlled variables.

iii) The model structure - i.e. how inputs and disturbances affect the measurements.

For the fixed bed reactor, the DYQUID in Figure 2 reveals that measurements of internal oxygen concentration and temperature contain information concerning all inputs whereas measurement of total mass flow rate e.g. at the reactor outlet only provides information about inlet flow rate. In addition, the DYQUID may be used for selection of suitable controls. The DYQUID in Figure 2 also reveals that the distributed forcing of inlet concentration and flow rate may be utilized to cancel disturbances already present in the process. Such an effect is not possible with inlet temperature which is a boundary forcing to a delay.

\section{Model Structure}

A qualitative model structure may be obtained from chemical engineering knowledge of qualitative process dynamics. Given the process controls and measurements it is possible to select a reasonably low number of parameters in the multivariable time series models described below. When a state of a quantity with predominantly convective flow is measured, all inputs which affect the particular quantity are identifiable. Using the DYQUID it is possible to investigate whether essential process inputs are qualitatively identifiable.

It is assumed that the input-output data from the chemical reactor can be described by a discrete-time multi-variable time-series model:

$$
\begin{aligned}
& x^{*}(t) a \\
& A\left(q^{-1}\right) x^{*}(t-1)+B\left(q^{-1}\right) u^{*}(t-d)+C\left(q^{-1}\right) e(t)
\end{aligned}
$$

where

$x^{*}(t)(\operatorname{dim} n)$ is model output, $x^{*}(t)=x(t)-x(t-1)$ where $x(t)$ is a vector of measurements

$u^{*}(t)(\operatorname{dim} m)$ is model input, $u^{*}(t)$ a $u(t)-u(t-1)$ where $u(t)$ is a vector of inputs

$e(t)(\operatorname{dim} n)$ is a zero means, white noise sequence d is the delay from input to output

$A\left(q^{-1}\right), B\left(q^{-1}\right)$ and $C\left(q^{-1}\right)$ are matrix polynomials of order_a, $b$ and $c$ in the backward shift opetor $q^{-I}$ i.e.

$$
\begin{aligned}
& A\left(q^{-1}\right)=A_{0}+A_{1} q^{-1}+\ldots+A_{a} q^{-a} \\
& B\left(q^{-1}\right)=B_{0}+\ldots+B_{b} q^{-b} \\
& C\left(q^{-1}\right)=I+C_{1} q^{-1}+\ldots+C_{c} q^{-c}
\end{aligned}
$$

The dimensions $(n, m, a, b, c)$ and the delay $d$ are assumed known and the matrices $A_{j}, i=0, \ldots, a ; B_{i}$, $i=0, \ldots, b$; and $c_{i}, i=1, \ldots, c$ are assumed unknown but constant or slowly varying.

Chemical processes are commonly subject to drift which by definition is slow. The phenomenon is reflected in the time series model equation (1) which has incremental states and controls. In this latter case the noise is coloured, but the influence of drift on the estimation is eliminated. In this case a control algorithm should be applied which eliminates offset.

The size of the model is defined when the measurements and controls are selected and $n, m, a, b, c$ and $d$ are fixed. If the model is not further structured it would be necessary to estimate $n(n(a+l+c)$ $+m(b+1)+1)$ parameters. However, using the DYQUID it is possible to reduce the above number of parameters significantly and still model the dominant thermal dynamics of the reactor and the influence of the inputs.

The measurements and controls are chosen from the qualitative process dynamics knowledge in the DYQUID (Figure 2). Since flow rate and rapid concentration measurements are only seldomly available in practice and the energy balance contains the significant capacity it is of interest to investigate the use of a number of temperature measurements. Here five equidistant sensors are used.

DYNAMIC QUANTITY INTERACTION DIAGRAM: (DYQUID)

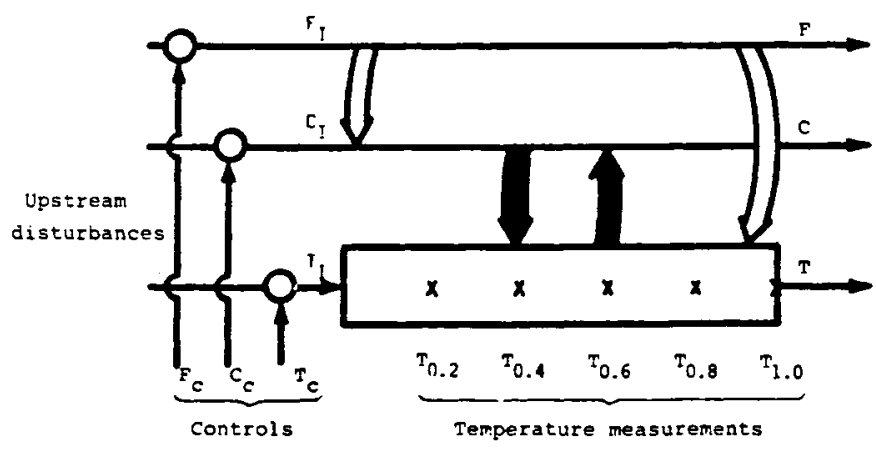

Fiqure 2:

Dynamic Quantity Interaction Diagram (DYQUID) for the fixed bed reactor. The set of temperature measurement positions considered in this paper is indicated by crosses. 
This choice makes it possible to propose a feasible model structure in addition to providing reasonably rapid measurement information about upstream disturbances both in upstream temperature which is a boundary forcing (through the temperature measurement at 0.2 ) and the distributed forcings as well as about the control object (Jorgensen et al. 1984). As controls the inlet temperature and most often one or two of the distributed forcings: inlet oxygen concentration and total flow rate are used. The distributed forcings have the advantage of being able to partially cancel disturbances already in the system.

Several model structures have been investigated earlier, Hallager and Jergensen (1981) and (1983). In this work a basic model structure for the above measurement set is selected. The termal delay time of the process at the nominal flow rate utilized in these experiments is $16 \mathrm{~min}$. For the example process the number of parameters is reduced using the DYQUID (Figure 2).:

i) The distributed disturbances (concentration and flow rate) influence all measurements with a delay of 1 sampling period (due to the sampling of the system).

ii) The thermal wave as observed by a number of temperature measurements is described essentially as a delay from the temperature input to the first measurement and from one measurement to the next. This behaviour necessitates a proper selection of sampling time in relation to model order.

The resulting model structure is based upon a sampling interval of $2 \mathrm{~min}$. and three $A$ and $B$ matrices. This sampling interval is approximately $20 \%$ larger than $1 / 10$ of the mean thermal residence time at the nominal operating point. The structure of $A_{0}$ and $B_{0}$ is:

$$
A_{0}=\left[\begin{array}{lllll}
x & 0 & 0 & 0 & 0 \\
x & x & 0 & 0 & 0 \\
0 & x & x & 0 & 0 \\
0 & 0 & x & x & 0 \\
0 & 0 & 0 & x & x
\end{array}\right] \quad B_{0}=\left[\begin{array}{lll}
x & x & x \\
0 & x & x \\
0 & x & x \\
0 & x & x \\
0 & x & x
\end{array}\right]
$$

and of $A_{i}$ and $B_{i}$ are:

$$
A_{i}=\left[\begin{array}{lllll}
0 & 0 & 0 & 0 & 0 \\
x & 0 & 0 & 0 & 0 \\
0 & x & 0 & 0 & 0 \\
0 & 0 & x & 0 & 0 \\
0 & 0 & 0 & x & 0
\end{array}\right] \quad \text { and } \quad B_{i}=\left[\begin{array}{lll}
x & 0 & 0 \\
0 & 0 & 0 \\
0 & 0 & 0 \\
0 & 0 & 0 \\
0 & 0 & 0
\end{array}\right] \quad i=1,2
$$

where $X$ denotes a parameter to be estimated. In addition to the delay term from one measurement to the next (subdiagonal elements), an auto-regressive term in each measurement is included in A (diagonal elements). This expansion is employed to partially account for dispersion. The first column of $B$ reflects the influence of inlet temperature and the following columns inlet concentration and flow rate respectively, the latter vectors are full according to i).
With the specified nominal delay and sampling interval the delay term from one measurement to the next is accounted approximately for by the subdiagonal elements in $A_{1}$. The subdiagonal elements in all three A-matrices are included in order to allow unknown flow rate variations within approximately $+100 \%$ and $-33 \%$ of the nominal flow rate. The structure of $B_{1}$ and $B_{2}$ follows from the convective nature of inlet temperature disturbances to the first measurement. If some controls are not applied the appropriate columns of $B_{i}$ are deleted for the actual basic model structure.

\section{Results}

All parameters were initially set to zero. After open loop reactor start-up, the identification was performed in open loop. During identification independent Pseudo-Random Binary Sequences were applied simultaneously to each actual input. When the model parameters had become nearly constant the PRBS generation was stopped. Now the loop was closed, and the controller allowed to bring the process to the desired steady state.

Finally, a step disturbance was introduced in the upstream temperature. Figures $3 a$ and $3 b$ show the closed loop response of selected axial temperatures and inlet conditions ( = control signal + upstream disturbance), at an inlet axygen concentration of 0.25 and 0.75 mole \% respectively. The experiment of $\mathrm{Fig}$. $3 b$ is somewhat hampered by noise around $5 \mathrm{~min}$, however, the intentioned disturbance is dominating. It is also noted that the flow rate input is not utilized in the model structure used for Fig. 3b, however, in the low inlet oxygen case (Fig. $3 a$ ) the process is not very sensitive to the flow rate, hence a comparison seems reasonable.

The qualitative picture of the inlet conditions is essentially the same for the two cases. After two samples the disturbance is detected and almost removed in the pertinent control variable ( $T_{\text {) }}$ ) over approximately 5 samples. The interesting point is the utilization of the oxygen input. In both cases it is lowered when the disturbance is detected. At the low oxygen operating point ( $\mathrm{Fig}$. $3 \mathrm{a}$ ) this action is reasonable as it cuts the maximum temperature deviation in the late part of the reactor. However, at medium oxygen ( $\mathrm{Fig}$. 3b) this action aggravates the inverse response. The reason for this undesirable behaviour is that the model structure does not include distant, fast effects of the temperature input. At this operating point the cross coupling between the thermal balance and the oxygen (mass) balance (see the DYQUID, Fig. 2) is sufficiently strong to induce fast effects of the temperature input. These effects are not accounted for in the model structure leading to the response shown.

Consequently, the model needs improvement to be valid at the medium oxygen concentration level. The DYQUID suggests faster effects further down the reactor. This could be included in the structure by including additional subdiagonals in the $A_{0}$ matrix and additional elements in the first column of $B_{0}$. 

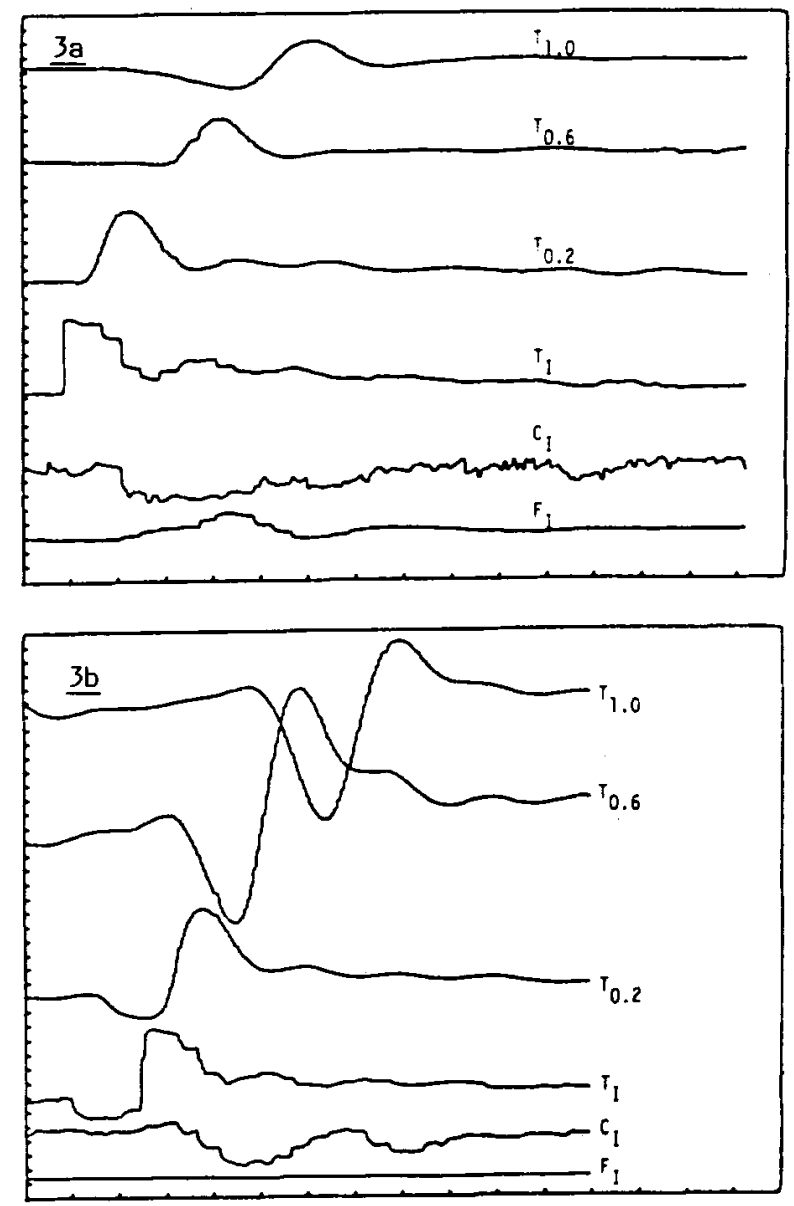

\section{Figure 3:}

Closed loop responses of selected axial reactor temperatures and inlet variables, subject to an upstream temperature step disturbance of height $5^{\circ} \mathrm{C}$, at inlet oxygen concentrations of $a$ : 0.25 and $b$ : 0.75 mole \% respectively.

\section{Conclusion}

Selection of a sufficiently flexible model structure is crucial for the performance of a multivariable self-tuning controller, when the controller is to work under different operating conditions.

It is demonstrated that the use of a simple graphical description of the dynamical elements of a chemical process (a DYQUID) may be used for predicting multivariable model structures. The examples shown demonstrate that the necessary structure complexity depends entirely upon the strength of the quantity interactions.

\section{References}

Bayoumi, M.M., K.Y. Wong and M.A. El-Bagoung (1981). A self-tuning regulator for multivariable systems. Automatica, 17, 575 .

Borison, U. (1975). Self-tuning regulators-industrial application and multivariable theory. Report 7513, Dept. of Automatic Control, LTH, Lund, Sweden.

Borison, U. (1979). Self-tuning regulators for a class of multivariable systems. Automatica, 15, 209-15.

Buchholt, F. and M. Kümmel (1981). A multivariable self-tuning regulator to control á double effect evap rator. Automatica, 17, 737.

Fortescue, T.R., L.S. Kershenbaum and B.E. Ydstie (1981). Implementation of self-tuning regulators with variable forgetting factors. Automatica, 17, 831-835.

Grimble, M.J. and T.J. Moir (1983). Multivariable weighted minimum variance self-tuning controllers. Preprints of IFAC Workshop on Adaptive Systems in Control and Signal Processing, Paper MIMO- 1 , San Francisco.

Hallager, L. (1984). Multivariable self-tuning control of a fixed-bed chemical reactor using structured. linear models. Ph.D. Thesis, Technical University of Denmark.

Hallager, L. and S.B. Jergensen (1981). Experimental investigation of self-tuning control of a gas phase fixed-bed catalytic reactor with multiple inputs. Paper No. 106.2 at $8^{\prime}$ th IFAC World Congress, Kyoto, Japan.

Hansen, K. (1973). Simulation of the transient behaviour of a pilot plant fixed-bed reactor. Chem.Eng. Sci., 28, 723-734.

Hansen, K. and S.B. Jergensen (1976). Dynamic modelling of a gas phase catalytic fixed-bed reactor. I-III. Chem.Eng.Sci., 31, 473-479 and 579-598.

Keviczky, L. and J. Hetthéssy (1977). Self-tuning minimum variance control of mimo discrete time systems. Automatic Control Theory Appl., 5, 11-17.

Peterka, V. and K.J. Aström (1973). Control of multivariable systems with unknown but constant parameters. Preprints of 3rd IFAC Symposium on Identification and System Parameter Estimation, p. 535-44. The Haoue, Netherlands.

Prager, D.L. and P.E. Wellstead (1980). Multivariable pole-assignment selftuning regulators. Proc. IEE, 12.8, part D, 1, 9-17.

Söderström, T., L. L jung and I. Gustavsson (1978). A theoretical analysis of recursive identification methods. Automatica, 14, 231-44. 